International Journal of Pure and Applied Mathematics

Volume 85 No. 6 2013, 1001-1008

ISSN: 1311-8080 (printed version); ISSN: 1314-3395 (on-line version)

url: http://www.ijpam.eu

doi: http://dx.doi.org/10.12732/ijpam.v85i6.3

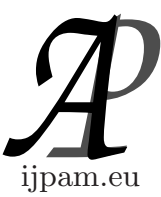

\title{
CONVOLUTIONAL CODES DERIVED FROM MELAS CODES
}

\author{
G.G. La Guardia \\ Department of Mathematics and Statistics \\ State University of Ponta Grossa \\ 84030-900, Ponta Grossa, PR, BRAZIL
}

\begin{abstract}
A family of convolutional codes is constructed algebraically in this note. These new convolutional codes are derived from Melas codes. The proposed codes have basic generator matrices, consequently, they are non catastrophic.
\end{abstract}

AMS Subject Classification: 81Q99

Key Words: convolutional codes, Melas codes, information theory

\section{Introduction}

Several papers addressing constructions of convolutional codes as well as their corresponding properties have been presented in the literature $[2,12,16,14$, $15,17,18,7,19,3,5,4,1,6,11]$. In [2], the author constructed an algebraic structure for convolutional codes; in [12], the author construct unit memory convolutional codes with good parameters. Constructions of maximum-distanceseparable (MDS) convolutional codes (in the sense that the codes attain the generalized Singleton bound introduced in [17, Theorem 2.2]), were presented in $[17,19,4]$. Concerning the optimality with respect to other bounds we have $[16,15]$. Strongly MDS convolutional codes were constructed in [3]. In $[18,7,1]$, the authors constructed convolutional BCH codes. In [5], doublycyclic convolutional codes were constructed and in [6], the authors described cyclic convolutional codes by means of the matrix ring. Finally, in [11], the author constructed families of convolutional codes derived from group character

Received: February 1, 2013

(c) 2013 Academic Publications, Ltd. url: www.acadpubl.eu 
codes.

In this note we construct algebraically convolutional codes derived from Melas codes by applying the well-known method introduced by Piret [14] and after generalized by Aly et al. [1, Theorem 3], which consists in the construction of convolutional codes derived from block codes.

The new convolutional codes have parameters

- $\left(q^{m}-1, q^{m}-m-1, m\right)_{q}$,

where $q \neq 2$ is a prime power, $q$ even, $n=q^{m}-1$ is the code length, $m \geq 2$, $k=q^{m}-m-1$ is the code dimension, $\delta=m$ is the degree of the code, and $d_{f} \geq 3$, where $d_{f}$ is the free distance of the code;

- $\left(2^{m}-1,2^{m}-m-1, m\right)_{2}$,

where $m$ is odd and $d_{f} \geq 5$.

The work is organized as follows. Section 2 presents a brief review on Melas codes and basic definitions and known results on convolutional codes. In Section 3, we present the contributions of the paper by constructing new convolutional codes derived from Melas codes and, in Section 4, a summary of the work is given.

\section{Preliminaries}

In this section we give necessary background for the proposed code construction.

Notation. We assume throughout that $p$ denotes a prime number, $q$ is a prime power and $\mathbb{F}_{q}$ is a finite field with $q$ elements. As usual, the parameters of a linear code are given by $[n, k, d]_{q}$, where $n$ is the code length, $k$ is the code dimension and $d$ is the minimum (Hamming) distance of the code.

\subsection{Melas Codes}

We assume that the reader is familiar with basics on cyclic codes. For details we refer to $[13,8]$. Recall that the Melas code $\mathbb{M}_{p}(m)$ is a cyclic code with parameters $[n, n-2 m, d \geq 3]_{p}$, where $p$ is a prime number and $n=p^{m}-1$ (see $[21,20])$. If $\alpha$ is a primitive element of $\mathbb{F}_{p^{m}}$ then a parity check matrix for $\mathbb{M}_{p}(m)$ is given by

$$
H=\left[\begin{array}{ccccc}
1 & \alpha & \alpha^{2} & \cdots & \alpha^{n-1} \\
1 & \alpha^{-1} & \alpha^{-2} & \cdots & \alpha^{-(n-1)}
\end{array}\right]
$$


considered as a matrix over $F_{p}$. This class of codes was generalized in [10] to $q$-ary alphabets, where $q$ is a prime power.

\subsection{Convolutional Codes}

The following background on convolutional codes can be found in $[9,8]$. We say that a polynomial encoder matrix $G(D) \in \mathbb{F}_{q}[D]^{k \times n}$ is basic if $G(D)$ has a polynomial right inverse. A basic generator matrix of a convolutional code $C$ is called reduced (or minimal $[19,8,6]$ ) if the overall constraint length $\delta=\sum_{i=1}^{k} \delta_{i}$ has the smallest value among all basic generator matrices of $C$; in this case $\delta$ is called the degree of the code. We define the weight of $\mathbf{v}(D) \in \mathbb{F}_{q}[D]^{n}$ as $\mathrm{wt}(\mathbf{v}(D))=\sum_{i=1}^{n} \mathrm{wt}\left(v_{i}(D)\right)$, where $\mathrm{wt}\left(v_{i}(D)\right)$ is the number of nonzero coefficients of $v_{i}(D)$.

Definition 1. [9] A rate $k / n$ convolutional code $C$ with parameters $(n, k, \delta)_{q}$ is a submodule of $\mathbb{F}_{q}[D]^{n}$ generated by a reduced basic matrix $G(D)=$ $\left(g_{i j}\right) \in \mathbb{F}_{q}[D]^{k \times n}$, that is, $C=\left\{\mathbf{u}(D) G(D) \mid \mathbf{u}(D) \in \mathbb{F}_{q}[D]^{k}\right\}$, where $\delta=\sum_{i=1}^{k} \delta_{i}$ is the degree, $\delta_{i}=\max _{1 \leq j \leq n}\left\{\operatorname{deg} g_{i j}\right\}, \mu=\max _{1 \leq i \leq k}\left\{\delta_{i}\right\}$ is the memory and $d_{f}=\operatorname{wt}(C)=\min \{w t(\mathbf{v}(D)) \mid \mathbf{v}(D) \in C, \mathbf{v}(D) \neq 0\}$ is the free distance of $C$.

The Euclidean inner product of two $n$-tuples $\mathbf{u}(D)=\sum_{i} \mathbf{u}_{i} D^{i}, \mathbf{v}(D)=$ $\sum_{j} \mathbf{u}_{j} D^{j}$ in $\mathbb{F}_{q}[D]^{n}$ is defined by $\langle\mathbf{u}(D) \mid \mathbf{v}(D)\rangle=\sum_{i} \mathbf{u}_{i} \cdot \mathbf{v}_{i}$. If $C$ is a convolutional code then its (Euclidean) dual code is defined by $C^{\perp}=\left\{\mathbf{u}(D) \in \mathbb{F}_{q}[D]^{n} \mid\right.$ $\langle\mathbf{u}(D) \mid \mathbf{v}(D)\rangle=0$, for all $\mathbf{v}(D) \in C\}$.

Let $[n, k, d]_{q}$ be a block code whose parity check matrix $H$ is partitioned into disjoint submatrices $H_{0}$ and $H_{1}$, each of which has $n$ columns and $\operatorname{rk} H_{0} \geq$ rk $H_{1}$, such that $H=\left[\begin{array}{ll}H_{0} & H_{1}\end{array}\right]^{T}$, obtaining the polynomial matrix $G(D)=$ $H_{0}+\tilde{H}_{1} D$, where $\tilde{H}_{1}$ is derived from $H_{1}$ by adding zero-rows to complete rk $H_{0}$ rows in total. The matrix $G(D)$ generates a convolutional code $V$ with $\operatorname{rk} H_{0}$ rows in total.

Theorem 2. [1, Theorem 3] Suppose that $C \subseteq \mathbb{F}_{q}^{n}$ is an $[n, k, d]_{q}$ code with parity check matrix $H \in \mathbb{F}_{q}^{(n-k) \times n}$ partitioned into submatrices $H_{0}, H_{1}$ as above such that $r k H_{0} \geq r k H_{1}$. Then $G(D)$ is a reduced basic generator matrix. Moreover, if $d_{f}$ and $d_{f}^{\perp}$ denote the free distances of $V$ and $V^{\perp}$, respectively, $d_{i}$ denote the minimum distance of the code $C_{i}=\left\{\mathbf{v} \in \mathbb{F}_{q}^{n} \mid \mathbf{v} \tilde{H}_{i}^{t}=0\right\}$ and $d^{\perp}$ 
is the minimum distance of $C^{\perp}$, then one has $\min \left\{d_{0}+d_{1}, d\right\} \leq d_{f}^{\perp} \leq d$ and $d_{f} \geq d^{\perp}$.

\section{The New Codes}

Most of known convolutional codes are constructed case by case or even by exhaustive computational search. In this light, we propose the construction of a family of convolutional codes derived from Melas codes by means of an algebraic method. The resulting codes are non catastrophic since they have basic generator matrices. We are now ready to show the main result:

Theorem 3. Let $q \neq 2$ be a prime power, $q$ even, and $n=q^{m}-1$, where $m \geq 2$. Then there exists an $\left(q^{m}-1, q^{m}-m-1, m\right)_{q}$ convolutional code with free distance $d_{f} \geq 3$.

Proof. We assume the same notation adopted in Subsection 2.2. Let $\mathbb{M}_{q}(m)$ be the Melas code of length $n=q^{m}-1$. Suppose that $\alpha$ is a primitive element of $\mathbb{F}_{q^{m}}$; then a parity check matrix for $\mathbb{M}_{q}(m)$ is given by

$$
H=\left[\begin{array}{ccccc}
1 & \alpha & \alpha^{2} & \cdots & \alpha^{n-1} \\
1 & \alpha^{-1} & \alpha^{-2} & \cdots & \alpha^{-(n-1)}
\end{array}\right],
$$

considered as a matrix over $F_{q}$. More precisely, let $\beta$ be any basis of $\mathbb{F}_{q^{m}}$ over $\mathbb{F}_{q}$. We expand all entries of $H$ with respect to $\beta$, resulting in a new matrix $H_{\mathbb{F}_{q}}$ over $\mathbb{F}_{q}$ having $2 m$ rows. Since $\mathbb{M}_{q}(m)$ has dimension $n-2 m$ it follows that $H_{\mathbb{F}_{q}}$ has rank $2 m$, so all rows of $H_{\mathbb{F}_{q}}$ are linearly independent. Further, we split $H_{\mathbb{F}_{q}}$ into disjoint submatrices $H_{0}$ and $H_{1}$ as follows:

$$
H_{\mathbb{F}_{q}}=\left[\begin{array}{c}
H_{0} \\
-- \\
H_{1}
\end{array}\right]
$$

where $\operatorname{rk} H_{0}=m=\operatorname{rk} H_{1}$. In other words, $H_{0}$ is derived from the matrix

$$
\left[\begin{array}{lllll}
1 & \alpha & \alpha^{2} & \cdots & \alpha^{n-1}
\end{array}\right]
$$

by expanding each entry with respect to the basis $\beta$, and $H_{1}$ is derived from the matrix

$$
\left[\begin{array}{lllll}
1 & \alpha^{-1} & \alpha^{-2} & \cdots & \alpha^{-(n-1)}
\end{array}\right]
$$

by expanding each entry with respect to $\beta$. From Theorem 2 and by construction, we can form the non catastrophic unit memory convolutional code $V$ of 
length $n=q^{m}-1$, generated by the matrix $G(D)=H_{0}+H_{1} D$. Since both matrices $H_{0}$ and $H_{1}$ have rank $m$, then $V$ has dimension $m$ and degree $m$.

Let us consider the (Euclidean) dual $V^{\perp}$ of the convolutional code $V$. We know that $V^{\perp}$ has length $n=q^{m}-1$, dimension $n-m$ and degree $m$. To compute the minimum distance $d_{f}^{\perp}$ of $V^{\perp}$, consider the cyclic code $C_{0}$ with parity check matrix $H_{0}$. Then it follows from the $\mathrm{BCH}$ bound that $C_{0}$ has minimum distance $d_{0} \geq 2$. The same logic applies to the cyclic code $C_{1}$ with parity check matrix $H_{1}$, so $d_{1} \geq 2$. It is easy to see that for $q \neq 2, q$ even, the minimum distance of the Melas code is $d \geq 3$. Applying again Theorem 2, it follows that $d_{f}^{\perp} \geq 3$. Therefore, one can get an $\left(q^{m}-1, q^{m}-m-1, m\right)_{q}$ convolutional code with free distance $d_{f} \geq 3$, as required.

In the case of binary convolutional codes of length $n=2^{m}-1$, with $m$ odd, one can derive convolutional codes having free distance $d_{f} \geq 5$, as shown in the following theorem.

Theorem 4. Consider that $n=2^{m}-1$, where $m \geq 3$ is an odd integer. Then there exists an $\left(2^{m}-1,2^{m}-m-1, m\right)_{2}$ convolutional code with free distance $d_{f} \geq 5$.

Proof. The proof follows the same line to that of Theorem 3. It is known that binary Melas codes of length $n=2^{m}-1$ for $m$ odd has parameters $[n, n-2 m, d \geq 5]$ (see [13, p. 206]). Assume that

$$
H=\left[\begin{array}{ccccc}
1 & \alpha & \alpha^{2} & \cdots & \alpha^{n-1} \\
1 & \alpha^{-1} & \alpha^{-2} & \cdots & \alpha^{-(n-1)}
\end{array}\right]
$$

is a parity check matrix for the code (considered as a matrix over $F_{2}$ ) and let $\beta$ be any basis of $\mathbb{F}_{2^{m}}$ over $\mathbb{F}_{2}$. Expanding all entries of $H$ with respect to $\beta$ one has a new matrix $H_{\mathbb{F}_{2}}$ over $\mathbb{F}_{2}$ with $2 m$ rows in total, each of which are linearly independent. We next split $H_{\mathbb{F}_{2}}$ into disjoint submatrices $H_{0}$ and $H_{1}$ as follows: $H_{\mathbb{F}_{2}}=\left[\begin{array}{c}H_{0} \\ -- \\ H_{1}\end{array}\right]$, where $\operatorname{rk} H_{0}=m=\operatorname{rk} H_{1}$, where $H_{0}, H_{1}$ are derived from

$$
\left[\begin{array}{lllll}
1 & \alpha & \alpha^{2} & \cdots & \alpha^{n-1}
\end{array}\right]
$$

and

$$
\left[\begin{array}{lllll}
1 & \alpha^{-1} & \alpha^{-2} & \cdots & \alpha^{-(n-1)}
\end{array}\right],
$$

respectively. By construction and based on Theorem 2, we can form the non catastrophic unit memory convolutional code $V$ of length $n=2^{m}-1$, dimension 
$m$ and degree $m$, generated by the matrix $G(D)=H_{0}+H_{1} D$. The (Euclidean) dual $V^{\perp}$ of $V$ has length $n=2^{m}-1$, dimension $n-m$ and degree $m$. Consider the code $C_{0}$ with parity check matrix $H_{0}$. Then one concludes from the $\mathrm{BCH}$ bound that $C_{0}$ has minimum distance $d_{0} \geq 3$ since the defining set of $C_{0}$ contains the sequence $\{1,2\}$ of two consecutive integers. The same justification applies to the cyclic code $C_{1}$ with parity check matrix $H_{1}$, since the defining set of $C_{1}$ contains the sequence $\{n-2, n-1\}$ of two consecutive integers; so $d_{1} \geq 3$. As was said previously, the minimum distance of $\mathbb{M}_{2}(m)$ satisfies $d \geq 5$. From Theorem 2 we have $d_{f}^{\perp} \geq 5$. Thus an $\left(2^{m}-1,2^{m}-m-1, m\right)_{2}$ convolutional code with free distance $d_{f} \geq 5$ can be constructed, and the proof is complete.

Example 5. If we consider $q=4$ and $m=2$ in Theorem 3, one obtains an $(15,13,2)_{4}$ convolutional code with free distance $d_{f} \geq 3$. Analogously, when $q=2$ and $m=3$, one obtains from Theorem 4 an $(7,4,3)_{2}$ convolutional code with free distance $d_{f} \geq 5$.

\section{Summary}

We have constructed a family of non catastrophic convolutional codes derived from Melas codes. These codes are constructed algebraically and not by computational search.

\section{Acknowledgments}

This research has been partially supported by the Brazilian Agencies CAPES and CNPq.

\section{References}

[1] S. A. Aly, M. Grassl, A. Klappenecker, M. Rötteler, P. K. Sarvepalli, Quantum convolutional BCH codes, e-print arXiv:quant-ph/0703113.

[2] G. D. Forney Jr, Convolutional codes I: algebraic structure, IEEE Trans. Inform. Theory, 16, No. 6 (1970), 720-738.

[3] H. Gluesing-Luerssen, J. Rosenthal and R. Smarandache, Strongly MDS convolutional codes, IEEE Trans. Inform. Theory, 52 (2006), 584-598. 
[4] H. Gluesing-Luerssen, W. Schmale, Distance bounds for convolutional codes and some optimal codes, e-print arXiv:math/0305135.

[5] H. Gluesing-Luerssen and W. Schmale, On doubly-cyclic convolutional codes, Applicable Algebra in Eng. Comm. Comput., 17, No. 2 (2006), 151170 .

[6] H. Gluesing-Luerssen and F-L Tsang, A matrix ring description for cyclic convolutional codes, Advances in Math. Communications, 2 No. 1 (2008), $55-81$.

[7] K. J. Hole, On classes of convolutional codes that are not asymptotically catastrophic, IEEE Trans. Inform. Theory, 46 No. 2 (2000), 663-669.

[8] W. C. Huffman and V. Pless, Fundamentals of Error-Correcting Codes, University Press, Cambridge (2003).

[9] R. Johannesson and K. S. Zigangirov, Fundamentals of Convolutional Coding, Digital and Mobile Communication, Wiley-IEEE Press (1999).

[10] A. Ketkar, A. Klappenecker, S. Kumar, and P. K. Sarvepalli, Nonbinary stabilizer codes over finite fields, IEEE Trans. Inform. Theory, 52, No. 11 (2006), 4892-4914.

[11] G. G. La Guardia, Convolutional Codes Derived From Group Character Codes, Submitted.

[12] L. N. Lee, Short unit-memory byte-oriented binary convolutional codes having maximum free distance, IEEE Trans. Inform. Theory, 22 (1976), 349-352.

[13] F. J. MacWilliams and N. J. A. Sloane, The Theory of Error-Correcting Codes, North-Holland (1977).

[14] Ph. Piret, Convolutional Codes: An Algebraic Approach, Cambridge, Massachusetts: The MIT Press (1988).

[15] Ph. Piret, A convolutional equivalent to Reed-Solomon codes, Philips J. Res., 43 (1988), 441-458.

[16] Ph. Piret and T. Krol, MDS convolutional codes, IEEE Trans. Inform. Theory, 29, No. 2 (1983), 224-232. 
[17] J. Rosenthal and R. Smarandache, Maximum distance separable convolutional codes, Applicable Algebra in Eng. Comm. Comput., 10 (1998), 15-32.

[18] J. Rosenthal and E. V. York, BCH convolutional codes, IEEE Trans. Inform. Theory, 45, No. 6 (1999), 1833-1844.

[19] R. Smarandache, H. G.-Luerssen, J. Rosenthal, Constructions of MDSconvolutional codes, IEEE Trans. Inform. Theory, 47, No. 5 (2001), 20452049 .

[20] H. Stichtenoth and C. Vo, Generalized Hamming weights of trace codes, IEEE Trans. Inform. Theory, 40 (1994), 554-558.

[21] J. Wolfmann, The weights of the dual code of the Melas code over GF(3), Discrete Math., 74 (1989), 327-329. 